\title{
Whole-organ atlas imaged by label- free high-resolution photoacoustic microscopy assisted by a microtome
}

Terence T. W. Wong, Ruiying Zhang, Hsun-Chia Hsu, Konstantin I. Maslov, Junhui Shi, et al.

Terence T. W. Wong, Ruiying Zhang, Hsun-Chia Hsu, Konstantin I. Maslov, Junhui Shi, Ruimin Chen, K. Kirk Shung, Qifa Zhou, Lihong V. Wang, "Whole-organ atlas imaged by label-free high-resolution photoacoustic microscopy assisted by a microtome," Proc. SPIE 10494, Photons Plus Ultrasound: Imaging and Sensing 2018, 104942N (19 February 2018); doi: $10.1117 / 12.2291056$ 


\title{
Whole-organ atlas imaged by label-free high-resolution photoacoustic microscopy assisted by a microtome
}

\author{
Terence T. W. Wong, ${ }^{\mathrm{a}, \mathrm{b}}$, Ruiying Zhang ${ }^{\mathrm{b}}$, Hsun-Chia Hsu, ${ }^{\mathrm{a}, \mathrm{b}}$, Konstantin I. Maslov ${ }^{\mathrm{a}}$, Junhui Shi ${ }^{\mathrm{a}}$, \\ Ruimin Chen $^{\mathrm{c}}$, K. Kirk Shung ${ }^{\mathrm{c}}$, Qifa Zhou ${ }^{\mathrm{c}, \mathrm{d}}$, and Lihong V. Wang, ${ }^{\mathrm{a}, *}$ \\ ${ }^{a}$ Caltech Optical Imaging Laboratory, Andrew and Peggy Cherng Department of Medical \\ Engineering, Department of Electrical Engineering, California Institute of Technology, Pasadena, \\ CA 91125, USA; \\ ${ }^{\mathrm{b}}$ Optical Imaging Laboratory, Department of Biomedical Engineering, Washington University in St. \\ Louis, St. Louis, MO 63130, USA; \\ ${ }^{\mathrm{C}} \mathrm{NIH}$ Resource Center for Medical Ultrasonic Transducer Technology, Department of Biomedical \\ Engineering, University of Southern California, Los Angeles, CA 90089, USA; \\ ${ }^{\mathrm{d}}$ Roski Eye Institute, Department of Ophthalmology and Biomedical Engineering, University of \\ Southern California, Los Angeles, CA 90089, USA;
}

\begin{abstract}
In biomedical imaging, all optical techniques face a fundamental trade-off between spatial resolution and tissue penetration. Therefore, obtaining an organelle-level resolution image of a whole organ has remained a challenging and yet appealing scientific pursuit. Over the past decade, optical microscopy assisted by mechanical sectioning or chemical clearing of tissue has been demonstrated as a powerful technique to overcome this dilemma, one of particular use in imaging the neural network. However, this type of techniques needs lengthy special preparation of the tissue specimen, which hinders broad application in life sciences. Here, we propose a new label-free three-dimensional imaging technique, named microtomy-assisted photoacoustic microscopy (mPAM), for potentially imaging all biomolecules with $100 \%$ endogenous natural staining in whole organs with high fidelity. We demonstrate the first label-free mPAM, using UV light for label-free histology-like imaging, in whole organs (e.g., mouse brains), most of them formalin-fixed and paraffin- or agarose-embedded for minimal morphological deformation. Furthermore, mPAM with dual wavelength illuminations is also employed to image a mouse brain slice, demonstrating the potential for imaging of multiple biomolecules without staining. With visible light illumination, mPAM also shows its deep tissue imaging capability, which enables less slicing and hence reduces sectioning artifacts. mPAM could potentially provide a new insight for understanding complex biological organs.
\end{abstract}

Keywords: Brain, photoacoustic imaging, microscopy, label-free, histology, microtome, whole-organ atlas

\section{INTRODUCTION}

Optical imaging techniques face a fundamental trade-off between tissue penetration and spatial resolution. Thus far, providing an image at organelle-level resolution of a whole organ has remained a challenge. Recently, optical microscopy assisted by chemical clearing or mechanical sectioning of tissue has been proven as a powerful technique to overcome this dilemma ${ }^{1-3}$. However, this type of techniques requires lengthy special preparation of the tissue specimen, hindering their applicability in life sciences. For example, diffusion staining of a whole brain ${ }^{1}$ is extremely slow due to the scant extracellular space in the central nervous system. Similarly, electrophoretic removal of lipids in the brain ${ }^{2}$, resulting in a transparent brain for easy staining and imaging, causes an uncertain loss of biological information. In addition, among all three-dimensional (3D) imaging techniques, histology is an attractive way to analyze specimens because histopathological interpretation can be readily applied from organelle to organ levels. Hence, finding a histology-like imaging method applicable to minimally processed tissue, ideally fresh tissue, can provide new insights into complex biological systems and make whole-organ microscopy a universal laboratory technique.

We propose a new label-free 3D imaging technique, microtomy-assisted photoacoustic microscopy (mPAM), for potentially imaging all biomolecules with $100 \%$ endogenous natural staining in whole organs with less sectioning and high fidelity. Photoacoustic (PA) microscopy (PAM) is a fast developing label-free imaging method. While in an

Photons Plus Ultrasound: Imaging and Sensing 2018, edited by Alexander A. Oraevsky, Lihong V. Wang, Proc. of SPIE Vol. 10494, 104942N · (c) 2018 SPIE · CCC code: 1605-7422/18/\$18 · doi: $10.1117 / 12.2291056$ 
unstained piece of tissue, most endogenous biomolecules do not fluoresce; however, all of them absorb photons at some wavelengths. Most absorbed light energy will be converted into heat, which results in an acoustic pressure rise propagating as ultrasound - the signal source for PA. PAM in reflection mode is applicable to large tissue volumes. Combined with a microtome for serial removal of previously imaged tissue sections, PAM performs well as a tool for imaging biomolecules of interest in an unstained organ at subcellular resolution ${ }^{4}$. Furthermore, PAM's label-free nature enables it to image differently embedded organs for different applications, e.g., paraffin and agarose are the most common embedding materials used in conventional histology and neuroscience, respectively. Here, we demonstrate the first label-free mPAM, using UV light for histology-like imaging without staining ${ }^{5}$, in whole organs (e.g., mouse brains), most of them formalin-fixed and paraffin- or agarose-embedded for minimal morphological deformation. Furthermore, mPAM with dual wavelength illuminations is also employed to image a mouse brain slice, demonstrating the potential for label-free imaging of multiple biomolecules. With visible light illumination, mPAM shows its deep tissue imaging capability, which enables less slicing and hence reduces sectioning artifacts.

\section{METHODOLOGY}

\subsection{Microtomy-assisted photoacoustic microscopy (mPAM)}

In mPAM (Fig. 1), an organ (e.g., a mouse brain), either formalin-fixed or fresh, and paraffin- or agarose-embedded, is mounted on an organ holder immersed in water. The tissue is automatically imaged under a computer control. A laser generates pulses at $266 \mathrm{~nm}$ wavelength (and $532 \mathrm{~nm}$ for dual wavelength illumination) to predominantly excite DNA/RNA in the tissue, and the generated PA waves are detected by a ring-shaped focused ultrasonic transducer. The sample holder is connected to a 3-axis motorized stage, which controls both the scanning for imaging and the tissue sectioning by the microtome. The mPAM system records and displays the cross-sectional images (e.g., coronal sections of a mouse brain) in real time during data acquisition. The exposed top tissue surface is imaged, then a thin layer is shaved off, and the new surface is imaged. This sequence is repeated to obtain a 3D image. The mPAM system currently provides a lateral resolution of $0.91 \mu \mathrm{m}^{4}$, more than sufficient to image individual cell nuclei without labeling. Moreover, our mPAM system can handle organs of various sizes because it is implemented in reflection mode.

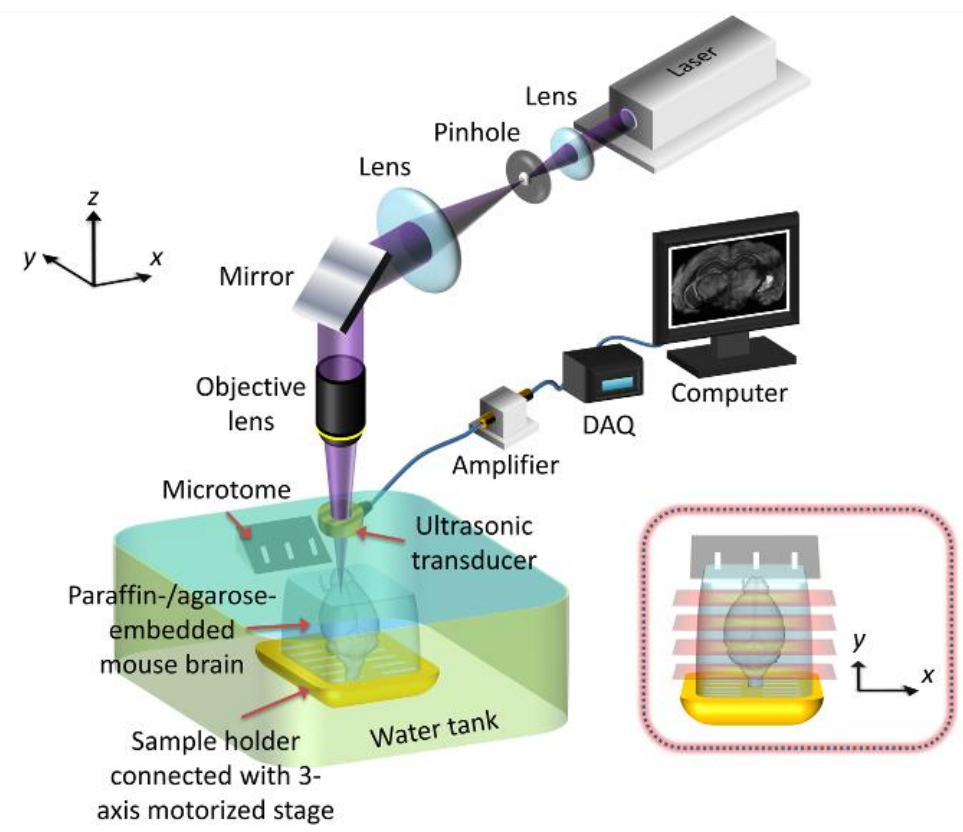

Figure 1. Schematic of the mPAM system for whole-organ imaging. The UV laser beam is spatially filtered and expanded by a pair of lenses and a pinhole. The beam is then focused through an objective lens and passed through a ring-shaped ultrasonic transducer onto the surface of the brain. The backward propagating acoustic waves are detected by the ringshaped ultrasonic transducer. The received acoustic pressure is transduced into an electric signal, amplified, and recorded by a data acquisition (DAQ). The bottom right inset shows the sectioning layers (red), which are all coronal sections. 


\section{RESULTS}

\subsection{Imaging a formalin-fixed paraffin-embedded mouse brain}

First, we validated the mPAM system by imaging a formalin-fixed thin paraffin section of a mouse brain (Fig. 2). The unstained paraffin section, fixed on a quartz slide that is UV transparent, was imaged by mPAM (Fig. 2a) and then stained with hematoxylin and eosin (H\&E) (Fig. 2b) for comparison with conventional histology. The corresponding close-up images are shown in Fig. 2c, d, respectively. The cell nuclei in the mPAM image were enhanced by Hessian filtering (Fig. 2a, c) and are highlighted in blue. The nuclei in the mPAM image match well with those in the H\&E image. Using the H\&E image as the gold standard, in identifying nuclei, mPAM has a sensitivity of $93.2 \%$, a specificity of $99.8 \%$, and a positive predictive value (PPV) of 96.7\%. This experiment shows that mPAM can pinpoint cell nuclei sensitively and specifically in a paraffin-embedded organ section.
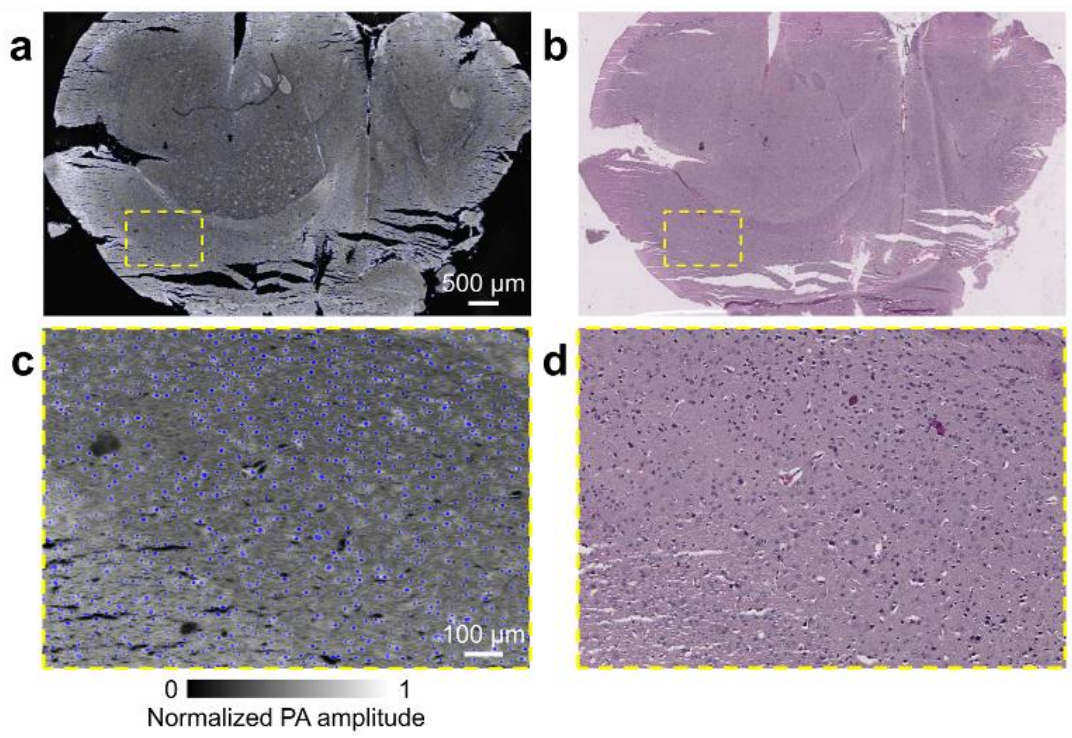

Figure 2. Imaging of a paraffin section of a mouse brain. (a) Label-free mPAM image, where the cell nuclei are enhanced by a Hessian filter and marked in blue. (b) Optical microscopy image acquired after H\&E staining. (c), (d) Close-up images of (a) and (b), respectively, corresponding to the yellow dashed regions in (a) and (b). The nuclei are clearly resolved by mPAM.

\subsection{Imaging formalin-fixed agarose-embedded mouse brains}

Second, we used agarose-embedded organs to further demonstrate applying mPAM to different embedding materials. The advantages of using agarose-embedding are two-fold: (i) Unlike paraffin, agarose is highly UV transparent and does not infiltrate into tissue ${ }^{6}$, which improves the imaging contrast of $\mathrm{mPAM}$, and (ii) agarose can be used for embedding fresh tissue, which enables mPAM to be readily used in life science studies. As an initial validation, a formalin-fixed paraffin-embedded mouse brain slice was imaged by mPAM and used as a reference (Fig. 3a). The slice was then deparaffinized, embedded in agarose, and re-imaged by mPAM (Fig. 3b). For comparison, an adjacent brain section was $\mathrm{H} \& \mathrm{E}$ stained and imaged by a conventional wide-field microscope (Fig. 3c). The corresponding close-up images are shown in Fig. 3d-f, respectively. The close-up images clearly show that a deparaffinized and agarose-embedded brain slice can reveal individual cell nuclei without any contrast enhancement algorithm, which was also validated with the H\&E close-up image. Paraffin is a UV absorbing material that infiltrates tissue. Thus, when it was washed out (i.e., deparaffinized), the background signal was reduced, and the contrast of all the original tissue structures was boosted (Fig. 3d, e). This experiment shows that with agarose embedding, mPAM can pinpoint cell nuclei without any contrast enhancement algorithm, further improving the accuracy of cell nuclear identification. 

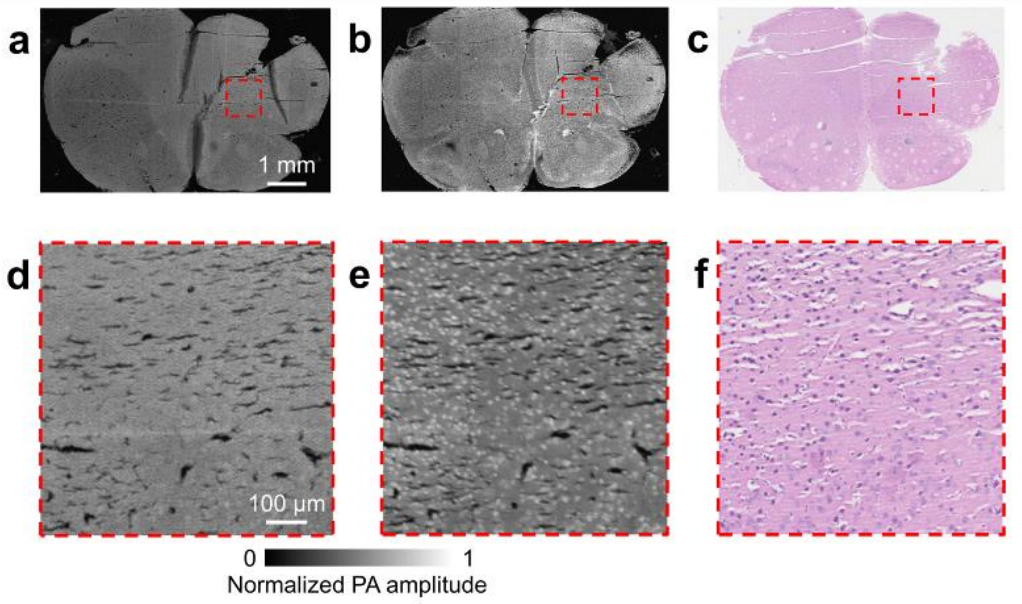

Normalized PA amplitude

Figure 3. Imaging of paraffin-embedded and deparaffinized sections of a mouse brain. (a) Label-free mPAM image of the paraffin section. (b) Label-free mPAM image of the deparaffinized section. (c) Optical microscopy image of the adjacent section after H\&E staining. (d)-(f) Close-up images of (a)-(c), respectively, corresponding to the red dashed regions in (a)(c). The mPAM image of the deparaffinized section shows cell nuclei clearly without resorting to image processing by Hessian filter.

As a proof of concept with a microtome, we imaged an entire formalin-fixed agarose-embedded mouse brain, with a $200 \mu \mathrm{m}$ section thickness. The imaged volume was $9.5 \mathrm{~mm}$ by $7.5 \mathrm{~mm}$ by $11.0 \mathrm{~mm}$. 10 representative coronal sections are shown in Fig. 4, and their corresponding positions are labeled on the 3D mouse brain model. To further show the strength of label-free mPAM that many biologically important features of the mouse brain could be imaged, two more formalin-fixed agarose-embedded mouse brains were imaged with different sectioning thicknesses, 300 and $400 \mu \mathrm{m}$. Together with the aforementioned $200 \mu \mathrm{m}$ sectioning thickness, we covered biological features that can be found in every 200, 300, and $400 \mu \mathrm{m}$. Fig. 5 shows a collection of images of features extracted from all agaroseembedded mouse brains. These features clearly reveal the unique capability of label-free mPAM, which allows imaging of different biomolecules that otherwise would require different labeling/dyes for simultaneous visualization. For instance, the rightmost mPAM images in Fig. 5 show cell nuclei, the olfactory limb, and blood vessels by imaging DNA/RNA, lipids, and hemoglobins with UV light illumination alone. Moreover, features such as myelinated axons and third ventricle can be imaged without staining due to their rich DNA/RNA and lipid contrasts (Fig. 5). By illustrating all the features with a single imaging modality and minimal tissue processing, mPAM enables understanding and exploring the structural or connection changes of different biological structures under different conditions, such as different diseases and stages, with high fidelity.
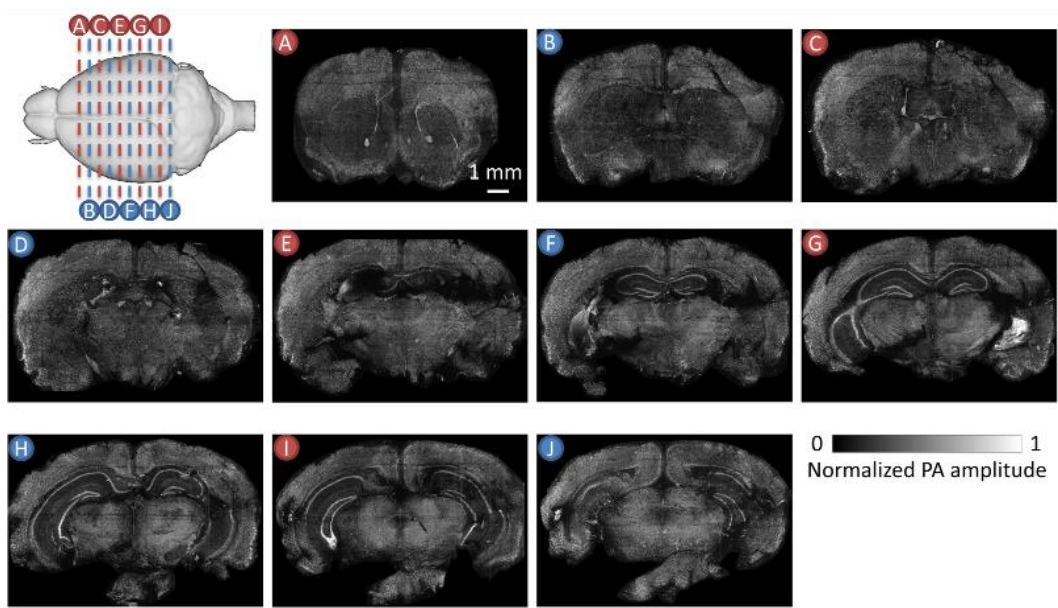

Normalized PA amplitude

Figure 4. 3D label-free mPAM image of an unstained mouse brain embedded in an agarose block. The top left inset shows a 3D mouse brain model. (A) $-(\mathbf{J})$ The relative positions of 10 coronal views are labeled in the 3D mouse brain model, and they are shown individually. 

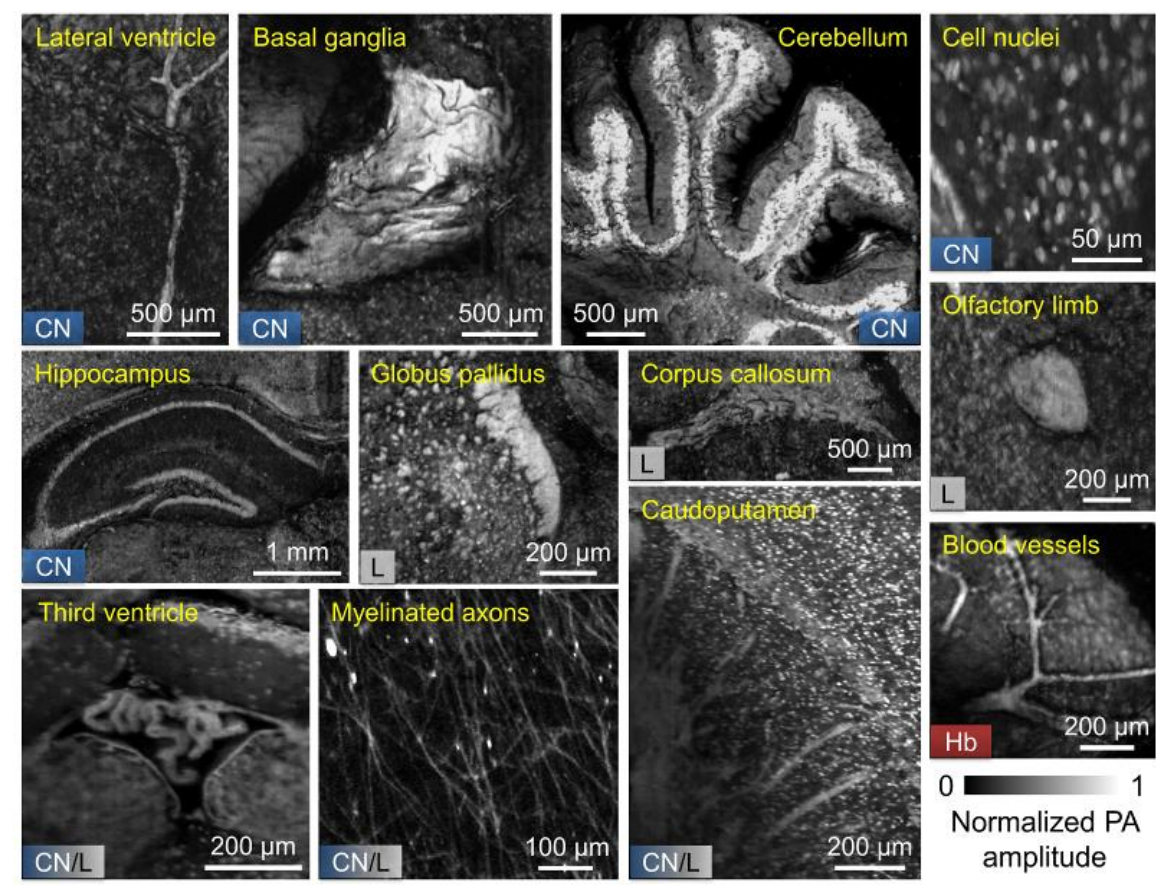

Offactory limb
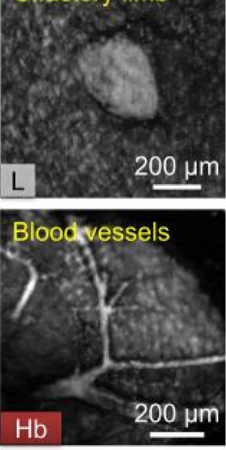

0 - 1

Normalized PA

amplitude

Figure 5. Image gallery of features extracted from label-free mPAM images of unstained mouse brains embedded in agarose blocks. All features are shown in coronal view. Collections of images showing the biomolecules that provide absorption contrast due to DNA/RNA (DR), lipids (L), and hemoglobins (Hb). Images of myelinated axons, third ventricle, and caudoputamen due to both DNA/RNA and lipids contrasts.

To show that mPAM can image more endogenous biomolecules, we used dual-wavelength illumination (266 and 532 $\mathrm{nm}$ ) to image an agarose-embedded brain slice (Fig. 6a, b). With $266 \mathrm{~nm}$ laser illumination, Fig. 6a shows mostly DNA/RNA and lipid contrasts, whereas with $532 \mathrm{~nm}$ laser illumination, Fig. 6b shows mostly cytochrome contrast. The overlay image (Fig. 6c) is displayed in two-channel pseudo colors, which represent the optical absorption color contrasts of the biomolecules at the two wavelengths and illustrates that more biomolecules are imaged by dual-wavelength mPAM than by single-wavelength mPAM.
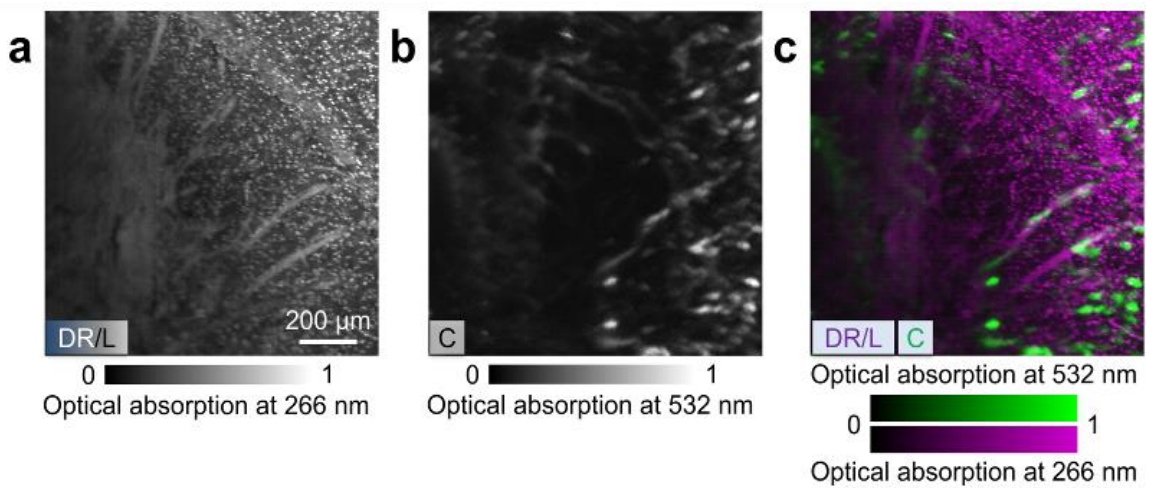

Figure 6. Imaging of an agarose-embedded mouse brain section by MPAM with dual wavelengths. (a) Label-free mPAM image with $266 \mathrm{~nm}$ laser illumination, which mostly shows DNA/RNA (DR) and lipid (L) contrasts. (b) Label-free mPAM image with $532 \mathrm{~nm}$ laser illumination, which shows cytochrome (C) contrast. (c) Overlay image of (a) and (b), where pseudo colors are used to illustrate the optical absorption color contrast of the biomolecules. 


\section{CONCLUSIONS}

To conclude, mPAM offers a new way to analyze disease-induced structural changes or the system function of a whole organ. By imaging cell nuclei and blood vessels, mPAM can also serve as a minimal-artifact substitute for histology. mPAM facilitates rapid 3D imaging of large tissue specimens. It can be readily applied to most standard paraffin blocks used in histology, e.g., paraffin blocks of an entire mouse brain. Such large volume registration-free histologic 3D imaging is impossible with any of the current choices for whole-organ microscopy. Moreover, mPAM can also be applied to fixed or fresh agarose-embedded tissue imaging, and so should find broad applications in basic life science studies.

\section{REFERENCES}

[1] Li, A. et al. "Micro-Optical Sectioning Tomography to Obtain a High-Resolution Atlas of the Mouse Brain," Science 330, 1404-1408 (2010).

[2] Chung, K. et al. "Structural and molecular interrogation of intact biological systems," Nature 497, 332-337 (2013).

[3] Amunts, K. et al. "BigBrain: An Ultrahigh-Resolution 3D Human Brain Model," Science 340, 1472-1475 (2013).

[4] Wong, T. T. W. et al. "Label-free automated three-dimensional imaging of whole organs by microtomy-assisted photoacoustic microscopy," Nat. Commun. 8, 1386 (2017).

[5] Wong, T. T. W. et al. "Fast Label-free multilayered histology-like imaging of human breast cancer by photoacoustic microscopy," Sci. Adv. 3, e1602168 (2017).

[6] Shim, K. "Vibratome Sectioning for Enhanced Preservation of the Cytoarchitecture of the Mammalian Organ of Corti," J. Vis. Exp. 17, 2793 (2011). 